La revue La revue pour l'histoire du CNRS

$2 \mid 2000$

Les premiers laboratoires du CNRS

Des hommes probables. De la procréation aléatoire à la reproduction normative

Jacques Testart, Seuil, Paris, 1999

Girolamo Ramunni

OpenEdition

Journals

Édition électronique

URL : https://journals.openedition.org/histoire-cnrs/378

DOI : 10.4000/histoire-cnrs.378

ISSN : 1955-2408

Éditeur

CNRS Éditions

Édition imprimée

Date de publication : 5 mai 2000

ISBN : 978-2-271-05708-2

ISSN : 1298-9800

Référence électronique

Girolamo Ramunni, « Des hommes probables. De la procréation aléatoire à la reproduction

normative », La revue pour I'histoire du CNRS [En ligne], 2 | 2000, mis en ligne le 06 mars 2006, consulté

le 20 mai 2021. URL : http://journals.openedition.org/histoire-cnrs/378 ; DOI : https://doi.org/10.4000/ histoire-cnrs.378

Ce document a été généré automatiquement le 20 mai 2021.

Comité pour l'histoire du CNRS 


\section{Des hommes probables. De la procréation aléatoire à la reproduction normative}

Jacques Testart, Seuil, Paris, 1999

Girolamo Ramunni

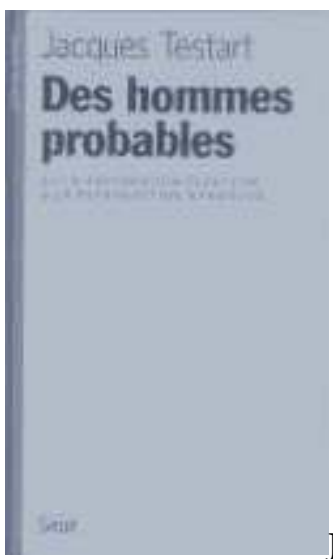

Jacques Testart a sans cesse fustigé les fausses assurances données pour la science dans ses écrits pour un public non spécialisé. Si, jusqu'à une date récente, la physique et la chimie occupaient le devant de la scène, actuellement, c'est plutôt la biologie et, plus particulièrement, ce qui touche à la génétique qui soulèvent des questions redoutables. En effet, la maîtrise de la procréation semble avoir atteint le stade où ce qui est incertain est transformé en probable, rejoignant ainsi au niveau des objectifs généraux le but déjà atteint par les autres champs du savoir. C'est la thèse centrale de ce livre, que l'auteur justifie en puisant dans l'histoire des vingt dernières années de recherche. Cette transition est explicite si l'on considère que l'on ne parle presque plus de procréation mais de reproduction.

1 Pour étayer sa thèse, Jacques Testart rappelle l'existence de situations paradoxales, nées du développement de la recherche. Il donne dès le début un exemple. Il informe le lecteur que « $40 \%$ de la production des pommes finit à la décharge, soit 124910 tonnes pour la région PACA» et que leur "fermentation présente un risque pour 
l'environnement, les acides organiques ainsi formés entraînant des éléments toxiques (fer, manganèse) dans la nappe phréatique » (p. 12). Pas de panique néanmoins, puisque les scientifiques sont là et étudient les remèdes éventuels. C'est une manière claire et efficace d'expliquer les situations qui peuvent se créer dans des cas qui, apparemment, semblent être très différents des pommes. Jacques Testart reconstruit de manière agréable et accessible à un lecteur non spécialiste les derniers développements de la génétique, de la biologie, de la bioéthique. Dans tout ce qu'il raconte, il se délecte à dénicher, dans les propos tenus par les scientifiques ou les journalistes, par les défenseurs inconditionnels des bienfaits de la science, les traces héritées d'une idéologie scientiste remontant au XIX ${ }^{e}$ et les mythes du progrès que ces discours entretiennent.

2 Prenant à contre-pied maintes affirmations, il montre qu'il faudrait se méfier de ces discours rassurants et " plutôt que craindre l'usage qu'un fou ou qu'un État totalitaire pourraient faire du bouturage humain, mieux vaut se préparer à refuser de prochaines justifications humanitaires ou thérapeutiques ». Car l'habituel discours du service rendu à l'humanité par la science et la vieille rhétorique qui fait de la science le seul moyen pour l'amélioration de la condition humaine ne sont que des moyens pour faire des hommes des "utilisateurs" acritiques par rapport au discours scientifique dominant. C'est pourquoi le changement dans les mots correspond à un changement bien plus profond. Quand on parle de reproduction plutôt que de procréation, on indique en même temps que l'on se place dans l'objectif de satisfaire le souhait de fabriquer l'homme que l'on désire. C'est là que se situe le changement essentiel apporté par la recherche. La reproduction apaise l'angoisse de l'homme qui veut échapper, à l'aide de la technique, à l'incertain de la procréation. Pour Jacques Testart, le chemin vers la «clonation » ne fait pas de doute, car ce sera la réponse recherchée au désir de tout prévoir même si ce n'est qu'avec un certain degré de probabilité. Jacques Testart n'est pas opposé à la science, il fait partie plutôt de ces scientifiques qui ne supportent pas tout ce qu'au nom de la science on fait croire aux gens qui n'ont pas une culture et une capacité critiques en ce domaine. Surtout, il lui semble insupportable que, la preuve ayant été faite que les règles que l'on se donne dans l'intérêt des citoyens sont systématiquement contournées, l'on continue à procéder de la même manière, en tenant toujours le même discours. Il rappelle en effet que, dans ces années de la génétique triomphante, il a été impossible de poser des règles a priori et de les faire respecter. D'où la conclusion qui soulève une question de fond: "Nous sommes forts puisque nous sommes les faiseurs de progrès, pensent les scientifiques. Il ne sera pas scientifiquement correct ni techniquement cohérent de dévoiler nos fragilités. Alors ils cautionneront la bioéthique qui dit la façon raisonnable de faire mais ils contesteront qu'il puisse être juste ou avisé de ne pas faire [...] L'une des plus grandes trahisons des hommes contre leur effort de civilisation, c'est la séparation récente des rôles par l'abandon aux uns des moyens objectifs de démonstration et aux autres des vertus culturelles du sens. Le mouvement de l'humanité, dès lors, entretient l'affrontement entre ces deux genres, plutôt que leur alliance dans la rencontre des êtres parlants. " Après avoir démontré à quel point les parents, les médecins, le marché et les gestionnaires de la société désirent que l'on produise des hommes probablement acceptables, Jacques Testart pose la question essentielle de la liberté par rapport à la science. Cela est possible si l'on retrouve le sens des choses, y compris de la recherche, et que l'on ne façonne pas des justifications de toutes sortes qui, en réalité, reviennent toutes à donner une priorité au résultat, quel qu'il soit, même si, pour le faire accepter 
au plus grand nombre, le seul moyen est de faire croire que l'objectif poursuivi est le mieux-être des utilisateurs.

AUTEUR

GIROLAMO RAMUNNI

Professeur à l'université de Lyon II 